\title{
Childhood polyarteritis nodosa: A rare presentation
}

\author{
Sandeep Lahiry, Shouvik Choudhury, Ayan Mukherjee, Koustuv Chowdhury \\ Post Graduate Trainee, Department of Pharmacology, Institute of Post Graduate Medical Education \& Research, \\ Kolkata, West Bengal, India
}

Polyarteritis nodosa (PAN) is a rare systemic vasculitis characterized by necrotizing arteritis of small to medium-sized arteries. It manifests as skin ulceration, hypertension, abdominal pain, digital gangrene, subcutaneous nodules etc. Childhood polyarteritis nodosa (CPAN) is a rare and often fatal disease. Clinically, CPAN often is part of the spectrum of Kawasaki disease (KD). There is no specific serological marker for diagnosis and confirmation is based on histopathology. Most common mode of pharmacological therapy includes corticosteroid or cyclophosphamide, along with antimicrobials for secondary infection prevention and plasmapheresis. Here, we present a case of a 16 year old boy with multiple skin ulceration, digital gangrene with few non-specific signs and symptoms, diagnosed as a case of CPAN, underwent regular therapy and follow-up.

\section{Access this article online}

Website:

http://nepjol.info/index.php/AJMS

DOI: 10.3126/ajms.v7i6.15724

E-ISSN: 2091-0576

P-ISSN: 2467-9100

Key words: Childhood polyarteritis nodosa, Corticosteroids, Mycophenolate mofetil

\section{INTRODUCTION}

Polyarteritis nodosa (PAN) is a multisystem, necrotizing vasculitis of small and medium-sized arteritis. It was first described by Kussmaul and Maier in1866. ${ }^{1}$ Common clinical presentations are lower extremity ulcerations, livedo-reticularis, subcutaneous nodules, digital gangrene, abdominal pain, hypertension or some non-specific features such as myalgia, malaise, weakness etc.

Childhood polyarteritis nodosa (CPAN) is a rare and often fatal disease. Polyarteritis has been described worldwide, although vasculitic diseases tend to be more common in individuals of Asian descent.

\section{CASE HISTORY}

A 16-year-old male patient of Indian origin, presented with past history of painful skin ulceration on back of trunk, shoulders and left gluteal region (Figure 1) for last 3weeks. Skin ulceration in various stages of evolution, from fresh to old scarred ones were evident. Dry gangrene was present on right index and middle fingers (Figure 2). There was no history of fever, cough, weight loss, dysuria, dyspnoea, sinusitis, testicular pain, seizure, long term smoking or recent drug intake prior to episode of skin lesions. No features of skin thickening or Raynaud's phenomenon were observed. No significant family history was present.

He had been suffering from similar symptoms since 2 years old. 3 months ago, he was admit under Rheumatology department with spontaneous onset erythematous skin rashes, gradually evolving into necrosis over inner-side of both thigh, inner-side of left arm and tip of right little finger for last 1 month.

He also had features of metabolic syndrome (obesity, insulin resistance, dyslipidemia) and delayed puberty during his last admission. Endocrine consultation was sought, and he was advised to take lowest possible dose of steroid.

On examination, his pulse rate was $90 /$ minute with normal volume and regular rhythm. Blood pressure was 110/70 mm of $\mathrm{Hg}$. He had cushingoid-habitus and acanthosis nigricans over nape of neck and axillary area. Systemic examination was unremarkable. Investigation 


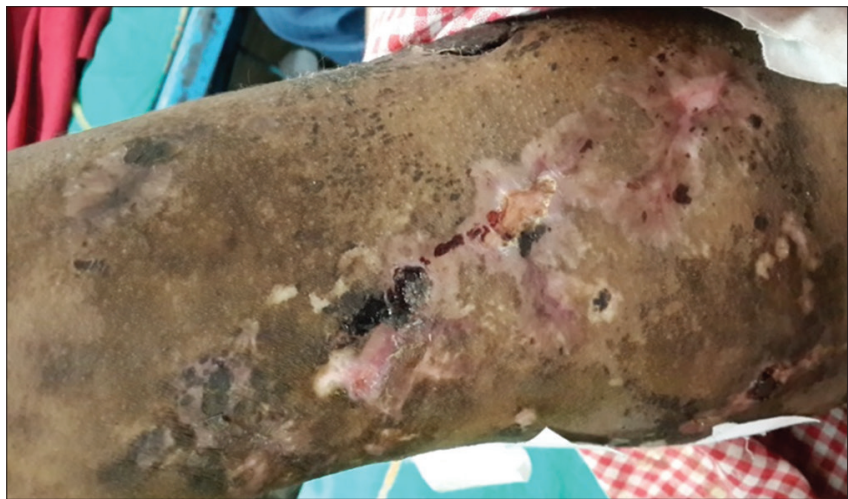

Figure 1: Photograph shows healing subcutaneous ulcer over thigh with scarring

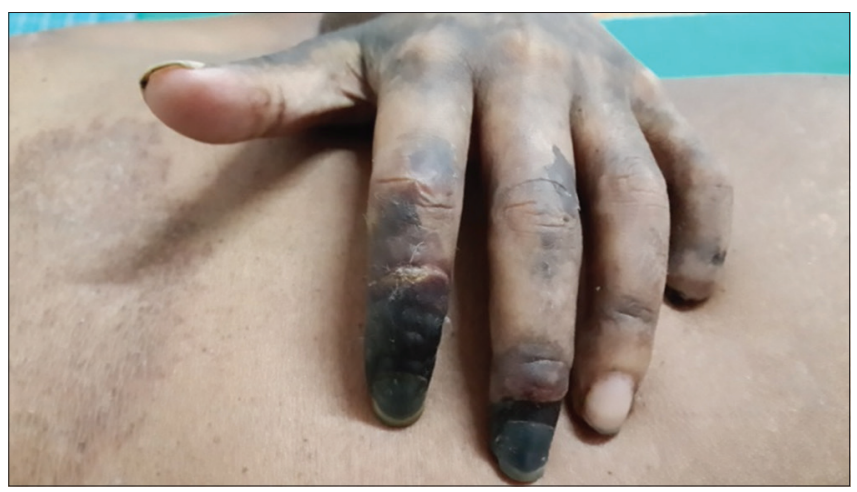

Figure 2: Healed gangrene of finger (i.e., autoamputated distal phalanx of little finger)

revealed Haemoglobin 8.8g/dl, Leucocyte count 19,300/ cumm with neutrophil $70 \%$, lymphocyte $26 \%$, eosinophil $2 \%$, monocyte $2 \%$; platelet $4.26 \times 10^{9} / \mathrm{L}$; serum bilirubin $0.6 \mathrm{mg} / \mathrm{dl}$, albumin $3 \mathrm{gm} / \mathrm{dl}$, globulin $3 \mathrm{gm} / \mathrm{dl}$, serum urea $26 \mathrm{mg} / \mathrm{dl}$, creatinine $0.6 \mathrm{mg} / \mathrm{dl}$, alanine aminotransferase 104IU/L, aspartate transaminase $45 \mathrm{IU} / \mathrm{L}$, alkaline phosphatase $106 \mathrm{IU} / \mathrm{L}$, serum sodium $141 \mathrm{mmol} / \mathrm{L}$, potassium $3.5 \mathrm{mmol} / \mathrm{L}$, calcium $10.1 \mathrm{mg} / \mathrm{dl}$, total cholesterol $144 \mathrm{mg} / \mathrm{dl}$, triglyceride $134 \mathrm{mg} / \mathrm{dl}$, high density lipoprotein $34 \mathrm{mg} / \mathrm{dl}$, serum luteinizing hormone $0.5 \mathrm{mIU} /$ $\mathrm{ml}$, follicle-stimulating hormone $0.3 \mathrm{mIU} / \mathrm{ml}$, serum iron $11 \mu \mathrm{g} / \mathrm{dL}$, total iron binding capacity $274 \mu \mathrm{g} / \mathrm{dL}$. All the serological markers Rheumatoid factor, Anti nucleotide antibody, Anti-neutrophil cytoplasmic antibody, Anticardiolipin antibody-IgM and IgG and lupus anticoagulant were negative. Hepatitis B surface antigen and Anti$\mathrm{HCV}$ were non-reactive. Ultrasound of abdomen was unremarkable except fatty changes in liver. Chest x-ray, echocardiography, ophthalmoscopy examinations were normal.

He first developed such features at 2years of age, which had responded to steroid therapy for 1-3months. There was polyarthalgia involving small joints of hands, proteinuria $(2.4 \mathrm{gm} /$ day $)$ without active sediments. Skin lesions used to recurr at intervals of 6-9months, after stopping steroid, for each episode.

Skin biopsy showed evidence of vasculitis of mediumsized vessels within dermis. Macroscopic and microscopic appearances of ulcers were like Cutaneous PAN (cPAN). As the child had digital gangrene, polyarthritis, proteinuria at the onset of disease, it fulfilled the criteria of CPAN. Currently, he is on oral prednisolone, mycophenolate mofetil, hydroxychloroquine therapy along with oral amoxicillin-clavulanic acid for secondary skin infection. Further approach is to include another immunosuppressant, oral cyclophospamide after stopping mycophenolate. Calcium and calcitriol supplementation have also been added.

\section{DISCUSSION}

PAN is a necrotizing arteritis of medium-sized vessels that has a predilection for involving the skin, peripheral nerves, mesenteric vessels (including renal arteries), heart, and brain can virtually involve almost any organ. ${ }^{2}$

For detecting a case of Childhood-PAN (CPAN), a systemic inflammatory disease with evidence of necrotizing vasculitis or angiographic abnormalities of medium or small-sized arteries (mandatory criterion) plus one of following five criteria: 1. Skin involvement; 2. Myalgia or muscle tenderness; 3. hypertension; 4. Peripheral neuropathy; 5. Renal involvement is to be fulfilled. ${ }^{3}$ Pharmacotherapy involves corticosteroids, systemic immunosuppressant like cyclophosphamide etc.

The involvement of kidney is usually limited and glomerulonephritis is not seen. This differs PAN from Microscopic Polyangitis where small vessel vasculitis cause glomerulonephritis and alveolitis. ${ }^{4}$

Clinically, CPAN often is part of the spectrum of Kawasaki disease (KD). CPAN with aneurysmal involvement of major coronary arteries and $\mathrm{KD}$ are clinically and pathologically indistinguishable. Indeed, the major distinction between $\mathrm{KD}$ and CPAN is that the diagnosis of $\mathrm{KD}$ is based entirely on clinical criteria, while the diagnosis of CPAN is based on histologic findings. Previously, it had been referred to as infantile polyarteritis nodosa (IPAN). The use of the term infantile is too restrictive, as infancy connotes age 1 year or younger.

Our patient presented with multiple skin ulceration with digital gangrene since childhood. He satisfied the required $3 / 10$ of the ACR criteria for diagnosis of PAN and Ozen's criteria. His diagnosis was confirmed by skin biopsy 
showing vasculitis of medium-sized vessels. Although skin ulcers responded to oral steroid, several recurrences occurred. So as steroid-sparing agent mycophenolate mofetil was introduced and dose of prednisolone was kept as low as possible. He had some non-specific complaints such as weakness, malaise, headache, and myalgia; but did not present with hypertension or haemorrhage which are usually seen in PAN. This case was also not associated with Hepatitis B infection.

Association of bacterial infections like streptococcal ones is described with recurrent gangrene in PAN. ${ }^{5}$ Other connective tissue diseases as childhood lupus and scleroderma may also cause gangrene. Despite being a rare disease, PAN is an important cause for gangrene and hypertension in children. ${ }^{4}$

Delayed or no treatment in PAN, may lead to significant morbidity and mortality. ${ }^{6}$ In our case, he is continuously on treatment and follow-up since childhood. Corticosteroids are mainstay of treatment on PAN. But to decrease the adverse effects of steroid in other systems, his dose of prednisolone was kept low and additional drugs are given. To conclude, CPAN is a challenging entity to diagnose and treat. Regular follow-up of patient is required.

\section{Key messages}

CPAN is a rare and often fatal disease affecting small and medium arteries. Overall prognosis is guarded.
The 10-year mortality rate, even in aggressively treated patients is high. Complications include digital necrosis, amputation, bowel infarction etc. Parent education and patient care should hence be coordinated by a multidisciplinary medical team.

\section{REFERENCES}

1. Kussmaul A MR. Über eine bisher nicht beschriebene Arterienerkrankung (Periarteritis nodosa), die mit Morbus Brightii und mit rapid fortschreitender allgemeiner Muskellähmung einhergeht. Dtsch Arch Klin Med 1866; 1: 484-518.

2. Lhote F, Cohen $\mathrm{P}$ and Guillevin L. Polyarteritis nodosa, microscopic polyangiitis and Churg-Strauss syndrome. Lupus 1998; 7:238-258.

3. Ozen S, Pistorio A, lusan SM, Bakkaloglu A, Herlin T, Brik R, et al. EULAR/PRINTO/PRES criteria for Henoch-Schönlein purpura, childhood polyarteritis nodosa, childhood Wegener granulomatosis and childhood Takayasu arteritis: Ankara 2008. Part II: Final classification criteria. Ann Rheum Dis 2010; 69:798-806.

4. Mogale KD and Shrivastava A. Report C. Childhood Polyarteritis Nodosa. A Clinical Diagnosis 2006; 43:911-913.

5. Albornoz MA, Benedetto AV, Korman M, McFall S, Tourtellotte CD and Myers AR. Relapsing cutaneous polyarteritis nodosa associated with streptococcal infections. Int J Dermatol 1998; 37:664-666.

6. Watanabe K, Rajderkar DA and Modica RF. A Case of Polyarteritis Nodosa Associated with Vertebral Artery Vasculitis Treated Successfully with Tocilizumab and Cyclophosphamide. Case Rep Pediatrics 2016; 2016:1-10.

\section{Authors Contribution:}

SL - Reviewed the literature and manuscript preparation; SC - Collected data, review of literature and helped in preparing first draft of manuscript;

AM - Literature search and critical revision of the manuscript; KC - Concept of study and review of study.

Source of Support: Nil, Conflict of Interest: None declared. 